\title{
Validation of the Meaning in Life Questionnaire (MLQ) in Chinese University Students and Invariance across Gender
}

\author{
Yongjie Jiang \\ School of Education, Nantong University, Nantong, China \\ Lei Bai \\ School of Science, Nantong University, Nantong, China \\ bl_007@ntu.edu.cn \\ Song Xue
}

State Key Laboratory of Cognitive Neuroscience and Learning \& IDG/McGovern Institute for Brain Research, Beijing Normal University, Beijing, China

\begin{abstract}
The goal of present study was to translate and validate the Meaning in Life Questionnaire (MLQ; Steger, Frazier, Oishi, \& Kaler, 2006) with a Chinese sample. We examined the internal consistency, validity and the factorial invariance across gender groups. A total of 347 Chinese university students were invited to take part in this study. The findings demonstrated that the scale had satisfactory internal reliability and corresponded to the theoretically proposed two-dimensional structure. Factorial invariance across males and females was first confirmed. The validity of the scale was supported by substantial correlations with existing measures of perceived stress, core self-evaluations, self-esteem, social support, positive affect, negative affect, life satisfaction. Overall, the current study provides initial evidence for the validity and reliability of a Chinese translation of the $M L Q$, and suggests that this scale can be used to measure meaning in life in Chinese university students.
\end{abstract}

Keywords: Meaning in life, Confirmatory factor analysis, reliability, validity.

\section{INTRODUCTION}

Meaning in life can be defined as "the sense made of, and significance felt regarding, the nature of one's being and existence" (Steger, Frazier, Oishi, \& Kaler 2006). The sense of meaning in life can be related to a wide range of life problems. Several studies have identified that there are psychological benefits such as happiness and well-being when individuals have a sense of purpose and meaning in life (e.g., King \& Napa 1998; Debats 1999; Scannell, Allen, \& Burton. 2002; King, Hicks, Krull, \& Del Gaiso, 2006; Steger \& Kashdan 2007). Recent studies have demonstrated that meaning in life is not only associated with psychological benefits, but may yield physical benefits because it is inversely associated with illicit drug and sedative use among male adolescents and with unsafe sex, binge drinking, and lack of exercise and diet control among females (Brassai, Piko, \& Steger, 2011).

To enable the study of individual differences in meaning in life, Steger et al. (2006) developed the 10-item Meaning in Life Questionnaire (MLQ), which consists of two scales to measure both the presence of meaning in life and the search for meaning in life. Exploratory factor analysis (EFA) identified this underlying two-factorial structure (i.e., Presence and Search); the results of the confirmatory factor analysis (CFA) with two independent samples provided support for the two-factor structure of the MLQ. The internal consistencies $(\alpha)$ ranged between .81 and .86 for Presence and .84 and .92 for Search. Other studies also provided support for the internal reliability of MLQ scores (e.g., Boyraz, Lightsey Jr, \& Can, 2013; Schulenberg, Strack, \& Buchanan, 2011). 
Moreover, a substantial amount of research has evaluated the concurrent and discriminant validity of the MLQ. Studies employing the MLQ have shown that the Presence subscale has been found to be positively correlated with extraversion, and agreeableness, optimism, life satisfaction, positive affect, happiness, gratitude, self-esteem, intrinsic religiosity, and positive life change, and negatively correlated with negative affect, neuroticism, depression, anxiety, posttraumatic stress, and materialism (Boyraz et al., 2013; Duffy \& Raque-Bogdan, 2010; Park, Park, \& Peterson, 2010; Steger et al., 2006; Steger, Oishi, \& Kashdan, 2009; Whittington \& Scher, 2010). For the Search subscale, correlations with other variables tend to be in the opposite direction. The Search subscale has been found to be positively correlated with anxiety, depression, negative affect (Park et al., 2010; Steger et al., 2009) and negatively correlated with the presence of meaning, life satisfaction and positive affect (Park et al., 2010; Steger et al., 2009). However, the link between Presence and Search seems more complicated than previously thought. For instance, the Search subscale was not related to the Presence subscale in American populations, whereas there was a negative correlation between these two subscales in Japanese populations (Steger et al. 2006; Steger, Kawabata, Shimai and Otake, 2008).

\subsection{The Current Study}

These results suggest that the MLQ is a reliable instrument to measure individual differences in the presence of meaning in life and the search for meaning in life. Although it has been translated into more than 20 languages for international use, because the MLQ is a relatively new instrument, few published studies have examined the reliability and validity of the MLQ in international samples (e.g., a American version, Steger et al., 2008; a Japanese version, Steger et al., 2008; a Spanish version, Steger, Frazier, \& Zacchanini, 2008; a Turkish Version, Boyraz et al., 2013). Our goal here was to develop and validate a Chinese version of the MLQ by testing whether the Chinese MLQ would replicate prior findings concerning factorial structure and relations to other constructs.

In general, gender invariance was always the first to be tested due to its basic nature for humans. Although the MLQ has been used in many studies, to our knowledge, no study has tested measurement invariance across gender groups so far. To make up the gap in literature for measurement invariance of the MLQ, we tested the factorial invariance of the MLQ across gender groups.

In addition, associations with a series of conceptually related constructs including positive affect, negative affect, life satisfaction, social support, core self-evaluations, self-esteem and perceived stress were examined, and predictions were formulated according to theoretical assumptions (Ryff \& Singer, 1998) and prior findings (Steger et al., 2006). We expected the presence of meaning in life to be associated with higher levels of social support, self-esteem, core self-evaluations, life satisfaction and positive affect and associated with low levels of negative affect and perceived stress. Correlations of the search for meaning in life with these variables were expected to be in the opposite direction.

\section{METHOD}

\subsection{Participants and Procedure}

A sample of 347 Chinese university students from southwestern China was recruited ( 223 females and 124 males; $64.3 \%$ and $35.7 \%$, respectively). Participant age ranged between 18 and 27 years (M $=20.95, \mathrm{SD}=1.41)$. Students represented all undergraduate classes $(17.3 \%$ freshmen, $40.3 \%$ sophomores, and $41.8 \%$ juniors), had a variety of majors (history, education, philosophy, physics, engineering, and agriculture). Written informed consent was obtained from all the participants prior to the study. 

across Gender

\subsection{Measures}

The Meaning in Life Questionnaire (Steger et al., 2006). The MLQ is a 10-item instrument with two subscales, Presence and Search. Each item is answered on a 7-point Likert type scale ranging from $1=$ absolutely untrue to $7=$ absolutely true. The Presence subscale includes five items that assess an individual's perception of the degree to which his or her life is meaningful, such as "I understand my life's meaning." The Search subscale assesses an individual's motivation to find meaning in life and consists of five items, such as "I am searching for meaning in my life." Separate Presence and Search scores are calculated with higher scores indicating higher levels of presence of meaning or search for meaning. Earlier research has shown good psychometric properties (Steger et al., 2006). For the current study, the MLQ was translated to Chinese, using the following procedure. First, the questionnaire was independently translated by two doctoral-level psychologists. Second, the two versions were compared and inconsistencies were resolved through consultation between the first author and the two translators. Third, the questionnaire was backtranslated into English by a bilingual psychologist. Finally, the original and backtranslated versions of the MLQ were checked for equivalence by the first author. Additionally, we also made several references to the Chinese translation of the MLQ provided by Steger and his colleagues.

The Satisfaction with Life Scale (SWLS, Diener, Emmons, Larsen, \& Griffin, 1985). The SWLS consists of five items. Each item is answered on a 7-point Likert type scale ranging from $1=$ strongly disagree to $7=$ strongly agree. It includes items such as, "I am satisfied with my life" and "In most ways my life is close to my ideal". Items are summed to form a general score of life satisfaction. The scale has been proved to have good validity and reliability in Chinese populations (e.g., Kong \& You, 2013; Song et al., 2013). In this study, the Cronbach alpha coefficient for the SWLS was .78.

The Positive and Negative Affect Schedule (PANAS; Watson, Clark, \& Tellegen, 1988). The PANAS consists of a word list describing two different affect states (10 positive and 10 negative), for example, excited and upset. Participants are instructed to indicate the extent they generally feel each affect using a 5-point Likert scale. Separate positive and negative affect scores are calculated with higher scores indicating participants feel more of that affect. The PANAS has good levels of reliability and validity (Kong \& Zhao, 2013). In this study, the Cronbach alpha coefficient was 0.84 for the Positive Affect Scale and 0.84 for the Negative Affect Scale.

The Rosenberg Self-esteem Scale (RSES; Rosenberg, 1965). The RSES is a 10-item self-report measure of global self-esteem. Each item is answered on a 7-point Likert type scale ranging from $1=$ strongly disagree to 4 = strongly agree. It includes items such as, "I am able to do things as well as most other people." and "I take a positive attitude toward myself". Scale scores are the sum of items with reverse coding of relevant items. The scores can range from 10 (low level of self-esteem) to 40 (high level of self-esteem). The RSES has good levels of reliability and validity (Kong, \& You, 2013; Kong, Zhao, \& You, 2012b, 2013). In this study, the Cronbach alpha coefficient for the RSES was .83.

The Core self-evaluations scale (CSES, Judge, Erez, Bono, \& Thoresen, 2003). The CSES is a 12-item scale developed in order to measure the underlying self-evaluative factor that is present across the four more specific traits of self-esteem, generalized self-efficacy, neuroticism and locus of control. It includes items such as, "I complete tasks successfully", and "Sometimes I feel depressed". Each item is answered on a 5-point Likert type scale ranging from $1=$ strongly disagree to $5=$ strongly agree. The Chinese version of the CSES has been found to be a reliable and valid measurement in assessing core self-evaluations in the Chinese adults (e.g., Kong, Wang, Zhao, 2014; Song et al., 2013; Sun et al., 2013). In this study, the scale had a Cronbach alpha coefficient of .71. 
The Multi-Dimensional Scale of Perceived Social Support (MSPSS: Zimet, Dahlem, Zimet, \& Farley, 1988). The MSPSS consists of 12 items relating to perceived social support, for example "My family really tries to help me', and "There is a special person who is around when I am in need". Each item is answered on a 7-point Likert type scale ranging from $1=$ strongly disagree to $7=$ strongly agree. Three separate scores can be calculated for the sources of support; Significant Other, Family and Friends. The MSPSS has good levels of reliability and validity (e.g., Kong et al., 2012a, 2013). In this study, the scale was internally consistent and had a Cronbach alpha coefficient of .80 .

The Perceived Stress Scale (PSS; Cohen, Kamarck, \& Mermelstein, 1983). This scale measures an individual's appraisal of their life as stressful (i.e. unpredictable, uncontrollable and overloading), consisting of 10 items. Item examples include, "How often have you felt nervous or stressed?" and "How often have you felt confident about your ability to handle your personal problems?" People rated how often they had experienced these feelings in the last month on a five-point Likert scale from 0 (never) to 4 (very often). Total scores range from 0 to 40 , with higher scores indicating greater overall distress. The PSS has good reliability and validity (e.g., Wang \& Chen, 2006). In this study, the scale was internally consistent and had a Cronbach alpha coefficient of .73.

\section{RESULTS}

\subsection{Confirmatory Factor Analysis}

AMOS Version 20 software (Arbuckle, 2009) was used to perform confirmatory factor analysis (CFA). Maximum likelihood estimation method and covariance matrices were analyzed in order to test the factor structure of MLQ. We elected to use multiple criteria for evaluating model misspecification, consisting of the goodness of fit index (GFI), comparative fit index (CFI), the root mean square error of approximation (RMSEA), and the standardized root mean square residual (SRMR). The following criteria were used to indicate goodness of fit: GFI and CFI .90 and higher, and RMSEA .10 or lower, SRMR .10 and lower (Byrne, 2001; Kline, 2005). The $\chi^{2}$ test statistic is reported, but because this value is inflated by sample size, our evaluation of model fit focused on other indices (Brown, 2006). The original two-factor structure of MLQ with 10 items proposed by Steger et al. (2006) was evaluated. Results of the CFA indicated an adequate model fit $\left[\chi^{2}(33)=134.71, p<.001\right.$; GFI $=.93$, $\mathrm{CFI}=.92, \mathrm{RMSEA}=.09$ and SRMR $=.08 \mathrm{l}$. The results of CFA are presented in Table 1 . The factor loadings ranged between .51 and .80 , and all factor loadings were substantial and significant $(p$ $<.001)$. The correlation between the two factors was .21 $(p<0.01)$.

Table1. Standardized factor loadings of the $M L Q$.

\begin{tabular}{|l|l|l|}
\hline Factors and items & Presence $^{\text {a }}$ & Search $^{\text {a }}$ \\
\hline 1. I understand my life's meaning. & .64 & \\
\hline 4. My life has a clear sense of purpose. & .78 & \\
\hline 5. I have a good sense of what makes my life meaningful. & .80 & \\
\hline 6. I have discovered a satisfying life purpose. & .78 & \\
\hline 9. My life has no clear purpose. & .51 & .71 \\
\hline 2. I am looking for something that makes my life feel meaningful. & & .56 \\
\hline 3. I am always looking to find my life's purpose. & & .78 \\
\hline 7. I am always searching for something that makes my life feel significant. & & .77 \\
\hline 8. I am seeking a purpose or mission for my life. & & .63 \\
\hline 10. I am searching for meaning in my life. & & \\
\hline
\end{tabular}

${ }^{a}$ All parameter estimates are significant $(p<.001)$. 
Validation of the Meaning in Life Questionnaire (MLQ) in Chinese University Students and Invariance across Gender

\subsection{Factor Invariance Across Males and Females}

CFA multiple group analyses were employed in order to examine whether the factor structure was invariant across gender (Byrne, 2004). Again, AMOS 20.0 was used. Two models were specified: In the first model, the factor loadings were constrained to be equal across gender; in the second model, the factor loadings were not constrained to be equal across both gender groups. The difference $\chi^{2}$ test between these two models was not significant, $\Delta \chi^{2}(8)=14.45, p>.05$. These results suggested that the measurement model for the MLQ was invariant across males and females (see Table 2).

Table2. Fit indicators across several models for the $M L Q$.

\begin{tabular}{|l|l|l|l|l|l|l|}
\hline Model & $\chi^{2}$ & $d f$ & RMSEA & SRMR & GFI & CFI \\
\hline Two-factor model & 134.71 & 33 & .09 &. .08 & .93 & .92 \\
\hline Invariance test for the model across gender & 166.76 & 66 & .07 & .09 & .92 & .92 \\
\hline No invariance constraints & 181.21 & 74 & .07 & .09 & .91 & .92 \\
\hline invariance constraints
\end{tabular}

Note. $R M S E A$ = root-mean-square error of approximation; SRMR = standardized root-mean-square residual; $C F I=$ comparative fit index; GFI = goodness of fit index.

\subsection{Internal Consistency Coefficient}

The internal consistency coefficients (Cronbach's $\alpha$ ) were calculated for the two-factor structure of MLQ with two independent samples. Cronbach's $\alpha$ for the Presence subscale was .82, for the Search subscale was .77, and for the MLQ scale was .76.

\subsection{Convergent and Discriminant Validity}

The convergent/discriminant validity of the MLQ was assessed by relating its subscales to measures of self-esteem, perceived stress, social support, life satisfaction, positive affect and negative affect. Means, standard deviations and correlations for all measures are presented in Table 3. As shown in Table 3, for the Presence subscale, Presence was substantially positively associated with self-esteem, core self-evaluations, social support, life satisfaction, and positive affect and substantially negatively associated with perceived stress and negative affect. For the Search subscale, Search was weakly associated with social support and positive affect and not associated with self-esteem, core self-evaluations, perceived stress, life satisfaction and negative affect. Moreover, we found that, compared to the Search subscale, Presence tended to have a stronger relationship with these constructs. These findings confirm the convergent and discriminant pattern of relations with these two meaning constructs.

Table3. Correlations and descriptive statistics for all variables

\begin{tabular}{|l|l|l|l|l|l|l|l|l|l|l|l|}
\hline & $\mathrm{M}$ & $\mathrm{SD}$ & 1 & 2 & 3 & 4 & 5 & 6 & 7 & 8 & 9 \\
\hline 1. Presence & 23.37 & 5.74 & 1 & & & & & & & & \\
\hline 2. Search & 25.30 & 5.35 & $.12^{*}$ & 1 & & & & & & & \\
\hline 3. Self-esteem & 30.11 & 4.53 & $.38^{* *}$ & .10 & 1 & & & & & & \\
\hline 4. Perceived stress & 27.88 & 4.97 & $-.36^{* *}$ & .001 & $-.51^{* *}$ & 1 & & & & & \\
\hline 5. Social support & 61.46 & 12.57 & $.30^{* *}$ & $.13^{*}$ & $.40^{* *}$ & $-.37^{* *}$ & 1 & & & & \\
\hline 6. Core self-evaluations & 37.32 & 5.96 & $.34^{* *}$ & .02 & $.56^{* *}$ & $-.57^{* *}$ & $.30^{* *}$ & 1 & & & \\
\hline 7. Life satisfaction & 17.78 & 5.81 & $.32^{* *}$ & -.07 & $.37^{* * *}$ & $-.41^{* *}$ & $.42^{* *}$ & $.43^{* *}$ & 1 & & \\
\hline 8. Positive affect & 30.18 & 6.42 & $.38^{* *}$ & $.24^{* *}$ & $.36^{* *}$ & $-.31^{* *}$ & $.29^{* *}$ & $.33^{* *}$ & $.31^{* *}$ & 1 & \\
\hline 9. Negative affect & 23.08 & 6.43 & $-.20^{* *}$ & .02 & $-.35^{* *}$ & $.53^{* *}$ & $-.30^{* *}$ & $-.34^{* *}$ & $-.25^{* *}$ & .05 & 1 \\
\hline
\end{tabular}

Note. $* p<.05, * * p<.001$. 


\section{DISCUSSION}

In the current study, the Chinese version of the MLQ was administered to a sample of 347 subjects with the aim of exploring the factor structure, gender invariance and validity of the MLQ. In line with numerous previous studies in different samples, the results of the analyses show that the MLQ is a reliable measure, in terms of good internal consistency. The results of CFA indicated that two-factor model revealed a better fit to the data, which suggests that this scale had better psychometric properties in Chinese sample.

One expending research for meaning in life in the present study was that the factorial invariance across gender groups was examined. For researches on meaning in life, difference in varying groups or time sets are always compared for diverse purposes. Thus, it is also meaningful to test the factorial invariance of the structure of meaning in life in Chinese culture. In present study, we found that there were no significant differences between constrained and unconstrained models, indicating that the factorial invariance of meaning in life does hold across gender groups. In other words, the structure of meaning in life does measure the same construct for different gender Chinese people. To our knowledge, this is the first study to demonstrate the factorial invariance across gender of the scale.

Interestingly, we found that the relationship between Search and Presence was found to be positive. This indicates that in Chinese individuals, although they have experienced the sense of meaning in life, they still need to search for meaning in life. These results support the previous findings that the link between Search and Presence is positive rather than negative in some collectivistic (e.g., Japan, Steger, et al., 2008) in comparison with individualistic cultures. This is probably because in some collectivistic cultures, particular philosophical beliefs and influences might affect individuals' ways of searching for, finding, and construing meaning.

Furthermore, validity of the MLQ was established by using a correlational inquiry. A Pearson correlation coefficient between subscales of MLQ (Presence and Search) and measures of self-esteem, perceived stress, social support, life satisfaction, positive affect and negative affect revealed satisfactory correlations. As expected, Presence was related to higher levels of self-esteem, social support, life satisfaction, and positive affect and related to lower levels of perceived stress and negative affect. The pattern of correlations is in accordance with the findings from other studies in Western countries (Boyraz et al., 2013; Duffy \& Raque-Bogdan, 2010; Park et al., 2010; Steger et al., 2006; Steger et al., 2009). However, in our study we did not found that Search had negative relations of with self-esteem, social support, life satisfaction, and positive affect and the positive relations with perceived stress and negative affect in western countries (Boyraz et al., 2013; Duffy \& Raque-Bogdan, 2010; Park et al., 2010; Steger et al., 2006; Steger et al., 2009). In our study, Search was weakly related to social support and positive affect and not related to self-esteem, core self-evaluations, perceived stress, life satisfaction and negative affect. These results are consistent with the study by Wang and Dai (2008). They found that Presence was not related to self-esteem, life satisfaction, negative affect and depression in Chinese young adults. These findings suggest that to some extent, searching for meaning in life is necessary for good health and well-being in the East cultures. To our knowledge, this is the first demonstrating the relations between meaning in life and related constructs, especially perceived stress, social support and core self-evaluations, and thus provide further validity of the MLQ in a Chinese sample.

In summary, the current study supports the two-factor model of the MLQ, and does confirm that the CSES is a reliable and valid instrument in the Chinese context to assess the meaning in life. Nevertheless, the sample of this study was composed of university students, so future studies examining applicability of MLQ on other populations (e.g., adolescents and olders) would be 
informative. Moreover, the results of the current study should be read cautiously because of its cross-sectional nature. The results of the study need to be cross validated with other methodologies and samples.

\section{ACKNOWLEDGEMENTS}

This research was supported by the Jiangsu Fund of Philosophy and Social Science in Higher Education (2014SJB605), Nantong University Project of Social Science (13W09). The authors declare no competing interests.

\section{REFERENCES}

Boyraz, G., Lightsey Jr, O. R., \& Can, A. (2013). The Turkish version of the Meaning in Life Questionnaire: Assessing the measurement invariance across Turkish and American adult samples. Journal of personality assessment, DOI:10.1080/00223891.2013.765882.

Brassai, L., Piko, B. F., \& Steger, M. F. (2011). Meaning in life: Is it a protective factor for adolescents' psychological health?. International journal of behavioral medicine, 18, 44-51.

Brown, T. A. (2006). Confirmatory factor analysis for applied research. New York: Guilford Press.

Byrne, B. M. (2001). Structural equation modeling with Amos: Basic concepts, applications and programming. Mahwah, NJ: Erlbaum.

Byrne, B. M. (2004). Testing for multi group invariance using AMOS graphics: A road less traveled. Structural Equation Modeling, 11, 272-300.

Cohen, S., Kamarck, T., \& Mermelstein, R. (1983). A global measure of perceived stress. Journal of health and social behavior, 24, 385-396.

Debats, D. L. (1999). Sources of meaning: An investigation of significant commitments in life. Journal of Humanistic Psychology, 39, 30-57.

Diener, E. D., Emmons, R. A., Larsen, R. J., \& Griffin, S. (1985). The satisfaction with life scale. Journal of personality assessment, 49, 71-75.

Duffy, R. D., \& Raque-Bogdan, T. L. (2010). The motivation to serve others: Exploring relations to career development. Journal of Career Assessment, 18, 250-265.

Kashdan, T. B., \& Steger, M. F. (2007). Curiosity and pathways to well-being and meaning in life: Traits, states, and everyday behaviors. Motivation and Emotion,31, 159-173.

King, L. A., \& Scollon, C. N. (1998). What makes a life good?. Journal of personality and social psychology, 75, 156-165.

King, L. A., Hicks, J. A., Krull, J. L., \& Del Gaiso, A. K. (2006). Positive Affect and the Experience of Meaning in Life. Journal of Personality and Social Psychology, 90, 179-196.

Kline, R. B. (2005). Principles and practice of structural equation modeling (2nd ed.). New York: Guilford Press.

Kong, F., Wang, X., \& Zhao, J. (2014). Dispositional mindfulness and life satisfaction: The role of core self-evaluations. Personality and Individual Differences, 56, 165-169.

Kong, F., \& You, X. (2013). Loneliness and self-esteem as mediators between social support and life satisfaction in late adolescence. Social Indicators Research, 110, 271-279.

Kong, F., \& Zhao, J. (2013). Affective mediators of the relationship between trait emotional intelligence and life satisfaction in young adults. Personality and Individual Differences, 54, 197-201. 
Kong, F., Zhao, J., \& You, X. (2012a). Social support mediates the influence of emotional intelligence on mental distress and life satisfaction in Chinese young adults. Personality and Individual Differences, 53, 513-517.

Kong, F., Zhao, J., \& You, X. (2012b). Emotional intelligence and life satisfaction in Chinese university students: The mediating role of self-esteem and social support. Personality and Individual Differences, 53, 1039-1043.

Kong, F., Zhao, J., \& You, X. (2013). Self-esteem as mediator and moderator of the relationship between social support and subjective well-being among Chinese university students. Social indicators research, 112, 151-161.

Park, N., Park, M., \& Peterson, C. (2010). When is the search for meaning related to life satisfaction?. Applied Psychology: Health and Well-Being, 2, 1-13.

Rosenberg, M. (1965). Society and the adolescent self-image. Princeton, NJ, USA: Princeton University Press.

Ryff, C. D., \& Singer, B. (1998). The contours of positive human health. Psychological inquiry, 9, $1-28$.

Scannell, E. D., Allen, F. C., \& Burton, J. (2002). Meaning in life and positive and negative well-being. North American Journal of Psychology, 4, 93-112.

Schulenberg, S. E., Strack, K. M., \& Buchanan, E. M. (2011). The meaning in life questionnaire: psychometric properties with individuals with serious mental illness in an inpatient setting. Journal of clinical psychology, 67, 1210-1219.

Song, G., Kong, F., \& Jin, W. (2013). Mediating effects of core self-evaluations on the relationship between social support and life satisfaction. Social indicators research, 114, 1161-1169.

Steger, M. F., Frazier, P., Oishi, S., \& Kaler, M. (2006). The Meaning in Life Questionnaire: Assessing the Presence of and Search for Meaning in Life. Journal of Counseling Psychology, 53, 80-93.

Steger, M. F., Frazier, P., \& Zacchanini, J. L. (2008). Terrorism in two cultures: Stress and growth following September 11 and the Madrid train bombings. Journal of Loss and Trauma, 13, $511-527$.

Steger, M. F., Kawabata, Y., Shimai, S., \& Otake, K. (2008). The meaningful life in Japan and the United States: Levels and correlates of meaning in life. Journal of Research in Personality, 42, 660-678.

Steger, M. F., Oishi, S., \& Kashdan, T. B. (2009). Meaning in life across the life span: Levels and correlates of meaning in life from emerging adulthood to older adulthood. The Journal of Positive Psychology, 4, 43-52.

Sun, P., Wang, S., \& Kong, F. (2013). Core self-evaluations as mediator and moderator of the relationship between emotional intelligence and life satisfaction. Social Indicators Research. DOI: 10.1007/s11205-013-0413-9.

Wang, S.Y., \& Chen, C.H. (2006). Psychosocial health of Taiwanese postnatal husbands and wives. Journal of Psychosomatic Research, 60, 307-363.

Wang, M.C., \& Dai, X.Y. (2008). Chinese Meaning in Life Questionnaire Revised in college students and its reliability and validity test. Chinese Journal of Clinical Psychology, 16, 459-461.

Watson, D., Clark, A., \& Tellegen, A. (1988). Development and validation of brief measures of positive and negative affect: The PANAS scales. Journal of Personality and Social Psychology, 54, 1063-1070.

Whittington, B. L., \& Scher, S. J. (2010). Prayer and subjective well-being: An examination of six different types of prayer. International Journal for the Psychology of Religion, 20, 59-68. 